\title{
A REMARK ON THE BOCHNER TECHNIQUE IN DIFFERENTIAL GEOMETRY ${ }^{1}$
}

\author{
H. WU
}

\begin{abstract}
It is observed that by pushing the standard arguments one step further, almost all the theorems in differential geometry proved with the help of Bochner's technique can be sharpened.
\end{abstract}

The main purpose of this note is to make an observation of a function-theoretic nature in global differential geometry. We shall take up a group of theorems centering around the Bochner technique [B], which typically shows that under the assumption of everywhere positive or negative curvature, certain geometrically interesting tensor fields (e.g., Killing, projective, etc.) vanish. Making use of the elementary fact that a nonconstant subharmonic function has no relative maximum, we shall show in essentially all cases that, instead of positivity or negativity, quasi-positivity or quasi-negativity of the Ricci curvature suffices, where by definition the Ricci curvature is quasi-positive iff it is everywhere nonnegative and is in addition positive (in all directions) at a point; similarly for quasi-negative. The main point of this observation is that this wholesale generalization of these theorems is achieved at no extra cost. Since the presence of zeroes of the Ricci (or any) curvature usually causes technical difficulties, such generalizations deserve notice. It therefore came as a surprise to the author that this one point seems to have been consistently overlooked in the standard literature.

To be specific, let us begin with the theorem in [B] that a Riemannian manifold $M$ with negative Ricci curvature has no nonzero Killing vector field whose length achieves a relative maximum. We now prove this assuming only quasi-negative Ricci curvature. (In particular, a compact Riemannian manifold with quasi-negative Ricci curvature has only a finite isometry group; cf. [Kb 2, p. 55] and [K-N, p. 251].) We follow the standard proof and obtain for any Killing field $X$ :

$$
\Delta\left(\frac{1}{2}|X|^{2}\right)=\sum_{i=1}^{n}\left|D_{V_{i}} X\right|^{2}-S(X, X)
$$

where $\Delta$ is the Laplace-Beltrami operator on functions, $|X|$ denotes the Riemannian norm of $X,\left\{V_{i}\right\}$ is an local orthonormal basis of vector fields, $D$ is the covariant differential operator and $S$ is the Ricci tensor [Kb 2, p. 56]. Note that the sum in (1) is globally defined. Since $S(X, X) \leqslant 0, \Delta\left(\frac{1}{2}|X|^{2}\right) \geqslant 0$ and $|X|^{2}$ is subharmonic. The assumption that $|X|$ has a relative maximum then implies $|X|$ is

Received by the editors February 5, 1979.

AMS (MOS) subject classifications (1970). Primary 53C20, 53C55.

${ }^{1}$ This research was partially supported by the National Science Foundation Grant MCS74-23180 and by the Miller Institute for Basic Research in Science of the University of California at Berkeley. 
a constant $c$. If $c>0, X$ is nowhere zero. Now at a point where the Ricci curvature is positive, the left side of (1) is zero while the right side would be positive. This contradiction shows $c=0$ and hence $X \equiv 0$.

The above reasoning is typical. In this special case of Killing fields, one could have concluded the proof in other ways. However, these are less adaptable to other situations than the preceding one.

This theorem of Bochner has extensions in the compact case to projective and conformal vector fields due respectively to Couty [C] and Yano [Yn 1], as well as a refinement due to Frankel [F]; we now discuss these. Couty's theorem states that on a compact Riemannian manifold of negative Ricci curvature, every projective vector field is zero. We shall prove it only assuming quasi-negative Ricci curvature. It suffices to consider an orientable $M$. Let $X$ be the given projective vector field, i.e., $X$ generates a one-parameter group of transformations of $M$ which preserve the geodesics in a set-theoretic sense. Since the Ricci tensor $S$ of $M$ is negative semidefinite, formula (19-3) on p. 209 of [C] implies $S(X, X)=0$ and $d X^{\#}=$ $\delta X^{\#}=0$, where $X^{\#}$ is the 1 -form dual to $X$ and $d$ and $\delta$ are the usual operators in Hodge theory (this requires compactness). In particular, $S(X, X)=\Delta X^{\#}=0$ where $\Delta X^{\#} \equiv-(d \delta+\delta d) X^{\#}$. Let $\left\{V_{i}\right\}$ be a local orthonormal basis of vector fields and define $D_{U V} \equiv D_{U} D_{V}-D_{D_{U} V}$. Then with $\langle$,$\rangle as the Riemannian metric,$ a standard calculation yields:

$$
\begin{aligned}
\Delta\left(\frac{1}{2}\left|X^{\#}\right|^{2}\right) & =|D X|^{2}+\left\langle\sum_{i=1}^{n} D_{V_{i} V_{i}} X^{\#}, X^{\#}\right\rangle \\
& =|D X|^{2}+S(X, X)+\left\langle\Delta X^{\#}, X^{\#}\right\rangle=|D X|^{2} \geqslant 0 .
\end{aligned}
$$

Hence $\left|X^{\#}\right|^{2}$ is subharmonic. Since $M$ is compact, $\left|X^{\#}\right|^{2}$ is constant and therefore $D X \equiv 0$, i.e. $X$ is parallel. In particular $X$ is Killing and the preceding theorem of Bochner implies $X \equiv 0$.

This result on the vanishing of projective vector fields on a compact $M$ with quasi-negative Ricci curvature is needed elsewhere [Wu] to establish the finiteness of the group of projective transformations. The latter requires a different set of ideas. This paper had its origin in attempting to obtain the preceding extension of Couty's theorem.

We now prove Yano's theorem in the form: a compact Riemannian manifold $M$ with quasi-negative Ricci curvature has no nonzero conformal vector field. Again it suffices to assume $M$ is orientable. Given a conformal vector field $X$ on $M$, let $X^{\#}$ be its dual 1-form. By [L, p. 128], we have $\Delta X^{\#}+(1-2 / n) d \delta X^{\#}=S(\cdot, X)$, where $n=\operatorname{dim} M \geqslant 2$. If [ , ] denotes the $L^{2}$ inner product on $M$, then

$$
\left[d X^{\#}, d X^{\#}\right]+\left[\delta X^{\#}, \delta X^{\#}\right]+\left(1-\frac{2}{n}\right)\left[\delta X^{\#}, \delta X^{\#}\right]=\int_{M} S(X, X)
$$

(cf. [C, p. 209]). Since by assumption $S(X, X) \leqslant 0$, this implies $S(X, X)=0$ and $d X^{\#}=\delta X^{\#}=0$. The proof in the projective case now applies verbatim.

Finally, Frankel's refinement of Bochner's theorem states that if $M_{0}$ is a compact Riemannian manifold with nonpositive sectional curvature and negative Ricci curvature and if $\varphi: M_{0} \rightarrow M_{0}$ is an isometry homotopic to the identity, then $\varphi$ is the 
identity. We shall prove this, with "negative Ricci curvature" replaced by "quasinegative Ricci curvature", again using the observation that a subharmonic function with a relative maximum is a constant. The noncompact analogue of this theorem [F, theorem on p. 375] will also be dealt with in due course. But first a preliminary discussion.

Let $M$ be a Cartan-Hadamard manifold, i.e., a simply-connected, complete Riemannian manifold with nonpositive sectional curvature. Given an isometry $\Phi$ : $M \rightarrow M$, its displacement function $f: M \rightarrow[0, \infty)$ is defined by $f(x)=[d(x, \Phi(x))]^{2}$, where $d$ denotes Riemannian distance; $f$ is a $C^{\infty}$ function. The Hessian of $f$ is $\left(D^{2} f\right)(X, Y) \equiv X Y f-\left(D_{X} Y\right) f$. To compute $D^{2} f(X, X)$ for a given $X \in M_{x}$, let $\alpha$ be the geodesic such that $\dot{\alpha}(0)=X$ and for all $s \in \mathbf{R}$ let $\gamma_{s}:[0,1] \rightarrow M$ be the geodesic joining $\alpha(s)$ to $\Phi(\alpha(s))$; denote $\gamma_{0}$ simply by $\gamma$. The following argument essentially follows p. 14 of [B-O]. (I am very grateful to B. O'Neill for his clarifying comments concerning this argument.) If $L(s)$ denotes the length of $\gamma(s)$, then $f(\alpha(s))=L(s)^{2}$ and $D^{2} f(X, X)=\left.\left(d^{2} / d s^{2}\right) L(s)\right|_{s=0} ^{2}$. Note that since for each $s,\left|\dot{\gamma}_{s}\right|$ is a constant, $L(s)^{2}=\left\{\int_{0}^{1}\left|\dot{\gamma}_{s}\right|\right\}^{2}=\int_{0}^{1}\left|\dot{\gamma}_{s}\right|^{2}$. Thus a straightforward argument yields:

$$
D^{2} f(X, X)=2 \int_{0}^{1}\left\{|\dot{X}(t)|^{2}-K(X(t))|X(t) \wedge \dot{\gamma}(t)|^{2}\right\} d t
$$

where $X(t)$ is the Jacobi field along $\gamma$ induced by the variation $\left\{\gamma_{s}\right\}, \dot{X}(t)$ denotes $D_{\dot{\gamma}(t)} X(t)$, and $K(X(t))$ denotes the curvature of the plane spanned by $\{X(t), \dot{\gamma}(t)\}$. Since $K(X(t)) \leqslant 0$ by assumption, (*) implies $D^{2} f(X, X) \geqslant 0$. This being true for all $X, f$ is a $C^{\infty}$ convex function ([B-O, Proposition 4.2]; see [B-O] and [G-W] for properties of convex functions). If $f(x) \neq 0$ but $D^{2} f=0$ on $M_{x}$, then for all $X \in M_{x}$ such that $X \perp \dot{\gamma}(0),(*)$ implies that $X(t)$ is parallel along $\gamma$ and that $K(X(t)) \equiv 0$. The former implies that $\Phi_{*}(X)$ (which is just $\left.X(1)\right)$ is perpendicular to $\dot{\gamma}(1)$ whenever $X \perp \dot{\gamma}(0)$, so that, in particular, $\Phi_{*}(\dot{\gamma}(0))=\dot{\gamma}(1)$; the latter implies that $S(\dot{\gamma}(t), \dot{\gamma}(t)) \equiv 0$ where $S$ is the Ricci tensor of $M$. Summarizing, we have:

LEMma. Let $M$ be a Cartan-Hadamard manifold. Then the displacement function $f$ of an isometry $\Phi: M \rightarrow M$ is convex. If for an $x, f(x) \neq 0$ but $D^{2} f=0$ on $M_{x}$, then $\Phi(\dot{\gamma}(0))=\dot{\gamma}(1)$ and $S(\dot{\gamma}(t), \dot{\gamma}(t))=0$ for $0 \leqslant t \leqslant 1$, where $\gamma:[0,1] \rightarrow M$ is the unique geodesic joining $x$ to $\Phi(x)$; if moreover $X(t)$ is the Jacobi field along $\gamma$ interpolating $X$ and $\Phi_{*}(X)$ where $X \in M_{x}$ and $X \perp \dot{\gamma}(0)$, then $X(t)$ is parallel along $\gamma$.

We can now prove Frankel's theorem very simply, assuming only quasi-negativity of the Ricci curvature. Notation and assumption as above, let $\pi: M \rightarrow M_{0}$ be the universal covering of $M_{0}$. Then $M$ is a Cartan-Hadamard manifold and the argument in [F] (see especially [Kb 2, pp. 57-59]) shows that there exists an isometry $\Phi: M \rightarrow M$ such that $\varphi \circ \pi=\pi \circ \Phi$ and that $d(x, \Phi(x))$ depends only on $\pi(x)$ for each $x \in M$. The displacement function $f$ of $\Phi$ may thus be considered a function on the compact $M$ and consequently achieves an absolute maximum. Since $f$ is convex by the Lemma, and hence subharmonic, $f$ reduces to a constant $c$. If $c>0$, let $y$ be a point of $M$ at which the Ricci curvature is negative. $D^{2} f$ being identically zero, the Lemma implies that $S(X, X)=0$ for the unit vector $X \in M_{y}$ 
tangent to the geodesic from $y$ to $\Phi(y)$. Contradiction. Hence $c=0$ and $\Phi$ is the identity; $\varphi: M_{0} \rightarrow M_{0}$ is the identity after all.

In the argument of the preceding paragraph, instead of a compact $M_{0}$, it suffices to have a complete $M_{0}$ such that the function $d(x, \Phi(x))$ considered as a function of $\pi(x) \in M_{0}$ achieves a relative maximum. This is the noncompact version of Frankel's theorem assuming only quasi-negative Ricci curvature (the theorem on $\mathrm{p}$. 375 of [F]).

The Lemma contains more information than that required for the proof of Frankel's theorem. The Appendix will extract from it a simple proof of an extension of Wolf's theorem [Wo].

In theory one can go on to re-examine all the results associated with the Bochner technique (cf., e.g., [L], [Yn 2]) from the present point of view. We choose to comment instead on three theorems. The first is the vanishing of the first Betti number of a compact Riemannian manifold with positive Ricci curvature [Yn 2, p. 37]. We now know that quasi-positive Ricci curvature in fact suffices. However, more general results can be proved by more elaborate arguments. On the one hand, Yau has proved the vanishing of the first cohomology group with compact support on a complete manifold when the Ricci curvature is quasi-positive [Yu 1, Theorem 6]. On the other, Cheeger and Gromoll have proved a structure theorem for compact manifolds of nonnegative Ricci curvature which implies that a compact manifold with quasi-positive Ricci curvature has a finite fundamental group [C-G, Theorem 3].

A second theorem to be discussed is the theorem in [K-W]. An usual, the reasoning in this note renders this theorem and all its corollaries valid also for quasi-positive or quasi-negative curvature. We single out three corollaries: (i) A compact Hermitian manifold $M$ with quasi-positive scalar curvature has no nonzero holomorphic $n$-form $(n=\operatorname{dim} M)$. (ii) A compact complex manifold with quasi-positive first Chern class, i.e., it can be represented by a $(1,1)$ form which is positive semidefinite everywhere and positive definite at a point, admits no nonzero holomorphic $p$-form, $p \geqslant 1$. (iii) A compact Hermitian manifold $M$ with quasinegative first Chern class has no nonzero holomorphic vector field. We note that, if positivity of the first Chern class is assumed in (ii), then the statement for $p=1$ can be strengthened to $M$ being simply-connected ([Kb 1] together with Yau's solution of the Calabi conjecture [Yu 2]). If in (iii) negativity of the first Chern class is assumed, then it can be strengthened to the finiteness of the automorphism group [Kb 2, p. 82]. Finally it should be remarked that, with a more elaborate argument, Yau [Yu 1, Theorem 5] has generalized the results of [K-W] to complete Kähler manifolds; in this regard, it is important to note that the results of [K-W] do not require the metric to be Kähler.

The most important among vanishing theorems is that of Kodaira; if $L$ is a positive line bundle on a compact complex manifold $M$, then $H^{q}\left(M, \Omega^{n}(L)\right)=0$ for $q \geqslant 1$. The results of this note suggest that $L$ should be assumed only to have a quasi-positive first Chern class; in this generality, the theorem is probably false. However, a much weaker statement has been conjectured for some years by 
Grauert and Riemenschneider (cf. [R 2, Conjecture II]), and the simple-minded arguments of this work are definitely inadequate for the settlement of this issue. What is true is that when $M$ is in addition assumed to be Kähler, then Kodaira's original argument [Kd] together with the reasoning above suffice to prove the "quasi-positive" Kodaira vanishing theorem without further ado (this has been done in the Appendix of [R 1], but by a different method). With the help of a theorem of Moišezon, one can then prove the Kodaira imbedding theorem for quasi-positive line bundles in a simple manner [R 2]. In particular, a compact Kähler manifold with quasi-positive or quasi-negative Ricci curvature is algebraic. We wish to call attention to these Kähler manifolds because they should be better understood from a differential geometric point of view.

Appendix.

Proposition. Let $M$ be a Cartan-Hadamard manifold and let $f$ be the displacement function of an isometry $\Phi: M \rightarrow M$ which is not equal to the identity. Then the following are equivalent:

(A) $f$ has a relative maximum.

(B) $f$ is a bounded function.

(C) $f$ is a positive constant.

(D) The vector field which at each $x \in M$ is tangent to the geodesic joining $x$ to $\Phi(x)$ and has norm $\sqrt{f(x)}$ is parallel.

Three remarks before the proof: (1) The equivalence of (B), (C) and (D) is the main theorem of [Wo]. Wolf made use of a rather technical result of Leon Green; the present proof (including (A)) requires only the Lemma above which is altogether elementary. (2) The equivalence of (A) and (B) unifies Wolf's theorem with Frankel's (specifically, the theorem on p. 375 on [F]), thereby settling a problem implicit in [F, p. 374, lines -9 to -7$]$. Perhaps more important than the formal unification is the underlying fact herein exposed that both theorems ultimately rest on the special properties of the displacement function. (In this regard, note that the function $|X|^{2}$ in the proof of Bochner's theorem is just an infinitesimal displacement function.) (3) If one considers continuous convexity, then it is easy to prove that $\sqrt{f}$ is a continuous convex function ([B-O, pp. 14-15]; cf. [G-W] for continuous convexity). Since a nonconstant convex function on $\mathbf{R}$ must have at least linear growth, another condition equivalent to (C) is the following: $\sqrt{f}$ has sublinear growth, i.e., for some $x \in M$ and for some $\alpha \in(0,1), \sqrt{f(y)} \leqslant(d(x, y))^{\alpha}$ for all $y \in M$.

Proof of Proposition. (A) $\Leftrightarrow(C)$. It is trivial that $(C) \Rightarrow(A)$. Conversely, a convex function being subharmonic, a nonconstant $f$ would have no relative maximum (cf. Lemma).

(B) $\Leftrightarrow(C)$. Trivially (C) $\Rightarrow(B)$. For the converse, take $x \in M$ and let $\zeta: \mathbf{R} \rightarrow M$ be any geodesic through $x$. Then $f \circ \zeta$ is a bounded convex function on $\mathbf{R}$ and hence constant. $\zeta$ being arbitrary, $f$ is constant.

$(C) \Leftrightarrow(D)$. (D) $\Rightarrow(C)$ because a parallel vector field has constant length. To prove $(\mathrm{C}) \Rightarrow(\mathrm{D})$, fix an $x \in M$ and let $\gamma:[0,1] \rightarrow M$ be the geodesic joining $x$ to 
$\Phi(x)$. Let $\Gamma: \mathbf{R} \rightarrow M$ be the maximal extension of $\gamma$. Since $f(x) \equiv c>0$ and $D^{2} f \equiv 0$, the Lemma implies $\Phi(\dot{\gamma}(0))=\dot{\gamma}(1)$. Thus $\Phi(\Gamma) \subset \Gamma$ and if $Y$ denotes the vector field in (D), then $Y \mid \Gamma=\dot{\Gamma}$. In particular $Y \mid \gamma=\dot{\gamma}$. Now take any unit vector $X \in M_{x}, X \perp \dot{\gamma}(0)$; it suffices to prove $D_{X} Y=0$. Let $\alpha$ be a geodesic such that $\dot{\alpha}(0)=X$. Let $r:[0,1] \times[0,1] \rightarrow M$ be the rectangle such that the curve $\gamma_{s}(t) \equiv$ $r(s, t)$ is exactly the geodesic joining $\alpha(s)$ to $\Phi(\alpha(s))$. The same argument as above then shows that $Y \mid \gamma_{s}=\dot{\gamma}_{s}$; thus $Y$ is the $t$-coordinate vector field of $r$. If $X^{*}$ denotes the $s$-coordinate vector field of $r$, then the last assertion of the Lemma states that $X^{*} \mid \gamma$ is parallel along $\gamma$. Note also that $X^{*}(x)=X$. Thus $D_{X} Y=$ $\left(D_{X^{*}} Y\right)(x)=\left(D_{Y} X^{*}\right)(x)=D_{\dot{\gamma}(0)} X^{*}=0$. Q.E.D.

Note (added March 20, 1979). The following paper has just come to the attention of the author: P. Gauduchon, Fibrés Hermitiens à endomorphisme de Ricci non négatif, Bull. Soc. Math. France 105 (1977), 113-140. One part of this paper also generalizes the vanishing theorems of $[\mathbf{K}-\mathbf{W}]$ to the case of quasi-positive or quasi-negative curvature (although without using this terminology). His method and viewpoint are different from those set forth in the present paper.

\section{BIBLIOGRAPHY}

[B-O] R. L. Bishop and B. O'Neill, Manifolds of negative curvature, Trans. Amer. Math. Soc. 145 (1969), 1-49.

[B] S. Bochner, Vector fields and Ricci curvature, Bull. Amer. Math. Soc. 52 (1946), 776-797.

[C-G] J. Cheeger and D. Gromoll, The splitting theorem for manifolds of nonnegative Ricci curvature, J. Differential Geometry 6 (1971), 119-128.

[C] R. Couty, Sur les transformations de variétés riemanniennes et kählériennes, Ann. Inst. Fourier (Grenoble) 9 (1959), 147-248.

[F] T. Frankel, On theorems of Hurwitz and Bochner, J. Math. Mech. 15 (1966), 373-377.

[G-W] R. E. Greene and $\mathrm{H}$. Wu, $C^{\infty}$ convex functions and manifolds of positive curvature, Acta Math. 137 (1976), 209-245.

[Kd] K. Kodaira, On a differential geometric method in the theory of analytic stacks, Proc. Nat. Acad. Sci. U.S.A. 39 (1953), 1263-1273.

[Kb 1] S. Kobayashi, On compact Kähler manifolds with positive Ricci tensor, Ann. of Math. 74 (1961), $570-574$

[Kb 2] _ Transformation groups in differential geometry, Ergebnisse der Mathematik und ihrer Grenzgebiete, Band 70, Springer-Verlag, Berlin, 1972.

[K-N] S. Kobayashi and K. Nomizu, Foundations of differential geometry, Volume 1, Wiley, New York, 1963.

[K-W] S. Kobayashi and H. Wu, On holomorphic sections of certain Hermitian vector bundles, Math. Ann. 189 (1970), 1-4.

[L] A. Lichnerowicz, Géométrie des groupes de transformations, Dunod, Paris, 1958.

[R 1] O. Riemenschneider, Characterizing Moisezon spaces by almost positive coherent analytic sheaves, Math. Z. 123 (1971), 263-284.

[R 2] _ A generalization of Kodaira's imbedding theorem, Math. Ann. 200 (1973), 99-102.

[Wo] J. A. Wolf, Homogeneity and bounded isometries in manifolds of negative curvature, Illinois $\mathrm{J}$. Math. 8 (1964), 14-18.

[Wu] H. Wu, Some theorems on projective hyperbolicity (to appear).

[Yn 1] K. Yano, On harmonic and Killing vector fields, Ann. of Math. 55 (1952), 38-45.

[Yn 2] K. Yano and S. Bochner, Curvature and Betti numbers, Ann. of Math. Studies, no. 32, Princeton Univ. Press, Princeton, N. J., 1953.

[Yu 1] S. T. Yau, Some function-theoretic properties of complete Riemannian manifolds and their applications to geometry, Indiana Univ. Math. J. 25 (1976), 659-670.

[Yu 2] _ On the Ricci curvature of a compact Kähler manifold and the complex Monge Ampere equation. I, Comm. Pure Appl. Math. 31 (1978), 339-411.

Department of Mathematics, University of California, Berkeley, California 94720 